\title{
A Study on the Innovation of E-commerce Service Mode under the Background of Big Data
}

\author{
Bo Liu \\ School of Foreign Languages, China West Normal University, Nanchong City, Sichuan \\ Province, 637002, China
}

Keywords: Big Data; E-commerce; Service Mode; Innovation

\begin{abstract}
With the development of information technology, the ability of data processing and analysis becomes more and more powerful in this information era. Data has penetrated into various industries, which becomes the significant factor of production. In the era of big data, e-commerce has also undergone great changes, and how to use big data to provide better service experience for the customer is the key point for future e-commerce service mode's innovation. Based on the author's learning and practical experience, this paper first analysed the e-commerce under the background of big data, then discussed the opportunity for e-commerce development under big data, and finally put forward the e-commerce innovation mode under the background of big data.
\end{abstract}

\section{Introduction}

In the current highly competitive market of e-commerce business, the technology of big data has its own extremely complex processing procedures, which is able to analyze the entire big data timely and precisely. It can provide the technical basis for effectively improving the e-commerce business market competitiveness, which can also use rational analysis of big data to develop more data information. In the era of big data, traditional e-commerce has also undergone great changes, and a large amount of information has been produced when the customers using the e-commerce, for which, the application of this part of data and innovation of service mode will be the inevitable trend under the background of big data.

\section{E-commerce under Big Data}

Under the background of bid data, e-commerce business can totally promote the value of e-commerce creation to precision, with the use of efficient big data collection, processing and analysis. E-commerce is generated with the behavior of enterprises and consumers in real time, whether in the e-commerce platform, mobile terminal or social media or any other third-party service platform, as long as these two parts intersect, e-commerce data will be generated. While traditional manipulation and analysis methods cannot process and use these massive data effectively. At the same time, e-commerce data also presents a high degree of diversity, which not only contains consumer reviews, feedbacks and various social information, but also has many personal information of consumers, such as the information of consumer location presented by mobile terminals. The data carrier includes text, picture, audio, video, and so on. As shown in Figure 1, taking the shopping website as an example, when you organize the collected big data and get consumer shopping intentions and behavior affecting factors, you can be more targeted to serve consumers, and persuade them to purchase your products. Going into the big data era, some garbage data, which considered to be useless previously, may provide more accurate and timely consumer information for e-commerce enterprises through big data analysis and cloud computing, and then it can provide the basis for enterprise marketing activities. In real 
life, Alibaba, Jingdong and other e-commerce enterprises just use the results of big data processing and analysis to carry out personalized recommendations and on-demand services.

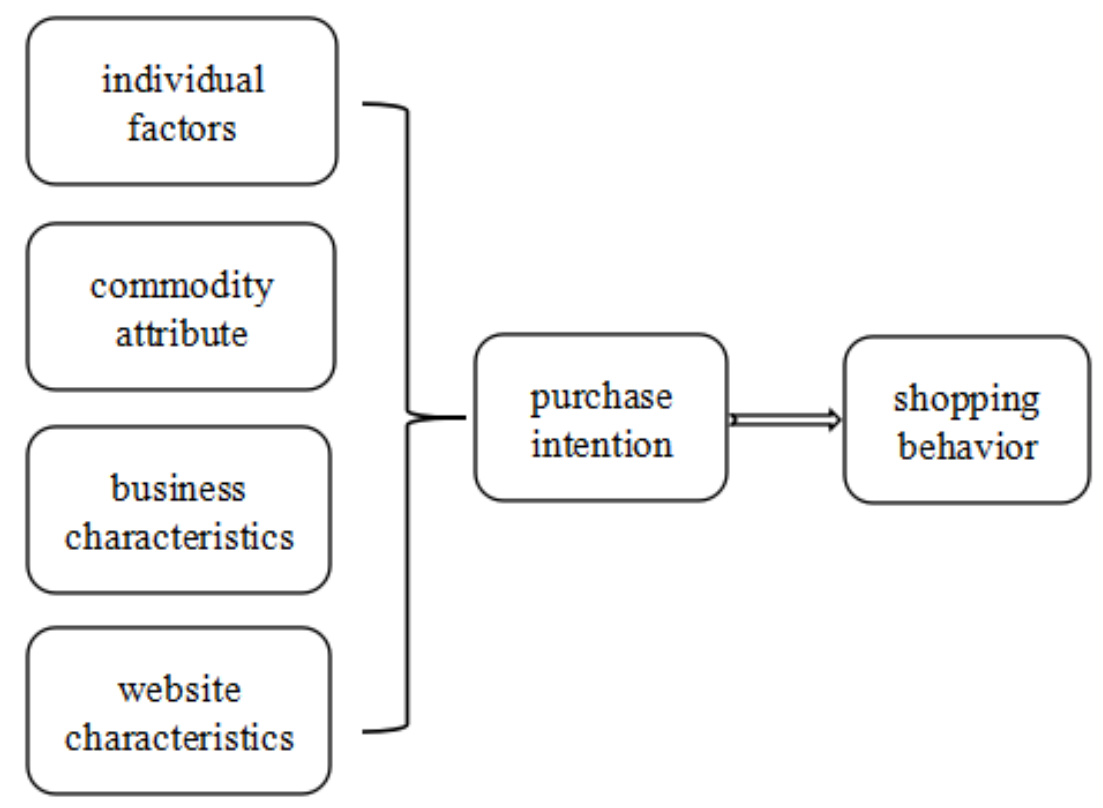

Fig.1 Effects of big data in shopping websites on consumer shopping intentions and behavior

\section{The opportunity for e-commerce development under big data}

Big data can better support the precision and real-time of e-commerce marketing. E-commerce development to today, its marketing platform and methods have undergone great changes. The use of e-commerce platform, mobile terminal, social network, internet and other equipment greatly increased the consumer data. While cloud computing, complex analysis system and other big data processing methods made it possible for people to integrate consumer data from various channels and generate useful marketing information. Compared with the traditional e-commerce data processing methods, big data processing methods are faster and more sophisticated, which help us greatly to analyze consumer preferences and consumption behavior trajectory scientifically.

Big data can better support the e-commerce highly differentiation and personalization. Under the background of traditional e-commerce marketing, enterprises and consumers are always in a state of two-way information asymmetry. On the one hand, the enterprise is difficult to understand the consumer behavior and consumer habits; on the other hand, the channel for consumers to understand the product information is relatively narrow. After entering the era of big data, companies can scientifically analyze massive amounts of data to gain more consumer information, then they can provide specific products and services for different consumers' need to maximize the satisfaction.

Big data can further promote the integration of value chain and supply chain. Big data and other new information technology can promote the integration of cross-area data from various channels, to make value chain and supply chain enterprises which surround the consumer behavior become an entirety. For example, big data can let enterprises of production, processing, marketing, warehousing, distribution, service and other parts form a dynamic alliance. These enterprises maybe from different geographical location and engaged in different industries, but they have the same purpose which is to meet the consumer demands. Through cooperation and creation, provide personalized products and services truly for consumers. 
Big data can promote the development of new value-added services mode. Various new information technologies are applied in the e-commerce, which generate a series of big data about production, consumption, finance, logistics. These big data belong to different fields can generate new fusions while being integrated used, resulting in the formation of new value-added services. On this basis, enterprises can also actively carry out similar financial credit services, supply chain integration and other value-added services. With the extensive application of big data, as well as the innovation of data analysis methods, it has produced a number of value-added services like internet finance, providing the new space for development for many small and medium enterprises, including e-commerce enterprises. In time, big data will spawn more new value-added service model, resulting in more industries.

\section{The e-commerce innovation mode under the background of big data}

Make on demand mode. In big data era, a prominent feature of e-commerce mode innovation is to identify and satisfy individual needs of consumers, that is, so-called make on demand mode. In this mode, all the work of e-commerce business surrounds the need of consumer, from design, development, production to the distribution of personalized products, consumers can participate in every step, to guarantee that consumers buy the products which they exactly prefer. In general, the make on demand mode has following characteristics: First, using e-commerce platform, social networks or mobile terminals and other diversified channels, to collect and obtain the full information of consumers, then excavate consumers' real and potential needs with the help of big data analysis or cloud computing technology. Second, after the acquisition of consumer potential demand, combined with the consumer demand preferences, they make personalized products and services on demand. Third, flexible production and value chain collaboration. That is, dynamically select the relevant enterprises from the value chain, and work together to produce products or provide services quickly, also automatically select the most suitable logistics and logistics channels in the value chain to meet consumer demand to the maximum extent. From the actual situation, the make on demand mode is still in the primary stage of development, with the further promotion and application of the cloud computing, big data and internet, make on demand mode will be able to develop more contents, and thus stimulate more consumption demand.

Deep fusion between online and offline mode. E-commerce value chain not only has the physical value chain, but also has the virtual one. With the increasing level of information utilization, the virtual value chain plays a more and more important role, and the trend of e-commerce value activity transforming from the entity value chain to the virtual value chain becomes more and more obvious. The combination of entity enterprises and e-commerce can achieve the development of both online and offline, but in different stages of development, the integration of online and offline is different, of which effect is also different. In the first stage, social commerce and mobile interactive terminals are applied in e-commerce, realize the integration of online and offline initially; in the second stage, consumer spending habits occur migration, online and offline integrate deeper. Consumers have been accustomed to online life in this stage, they mostly prefer online consumption; in the third stage, online and offline resources appear to be fully integrated. Online and offline deep integration mode responses to the current consumer habits and living environment, e-commerce enterprises should make use of consumers' fragmented time, for which they develop more products and provide better service to meet the consumer experience needs. Entity enterprises should take the initiative to move closer to the virtual value chain, under the support of big data analysis and processing technology, strengthen the enterprise logistics warehousing function, surround the personalized service, achieve inventory optimization, and effectively reduce the cost of enterprise inventory. 
Internet finance and online supply chain finance. Internet finance is the new thing that has emerged in recent years. Its foundation is also the integration of various data ports such as consumers, e-commerce enterprises, logistics and finance. In the platform of e-commerce, time and space constraints will no longer be a problem, bid data analysis and processing technology allows both sides of the trade to get information easily, which achieves direct docking and forming cooperation. For most small and medium enterprises, because of internet finance using e-commerce platform, big data analysis and processing, cloud computing and other technologies, the costs of two sides match, trading and risk sharing are greatly reduced, which ease the small and medium enterprises' financing difficulties to a large extent. At present, the internet lending services and similar companies have sprung up. Some e-commerce platform also put cloud computing, big data technology into use of information flow, business flow, fund flow, logistics integration, and provide network supply chain financial services. This kind of financial services can better integrate the internet and big data, achieving the seamless docking between enterprise information system and the electrical business platform, for which data and resource sharing more powerful, e-commerce platform learning enterprise information more timely and real. Trading behavior becomes more convenient, fund security becomes more guaranteed, through the establishment of third-party supervision and settlement system, it can effectively protect the security of both buyers and sellers. It is worth pointing out that the online supply chain using the entire supply chain information, which is also the integration of entire supply chain logistics, information flow and capital flow. Through the effective cooperation of the supply chain, it can largely reduce the operating costs of enterprises, thus showing a more powerful competitiveness.

\section{Summary}

Based on the big data technology support, e-commerce personalized information service mode has the characteristics of independency. It can provide consumers with personalized shopping service recommendation service system, and has further optimization on the basis of this mode technology, which performs detailed basic work processing aimed to optimize the content. This also indicates that the mode is feasible in the development of existing e-commerce, and its humanized, intelligent user-oriented service mode truly meets the current trend of the times of big data and cloud computing, it is the future direction of enterprise innovation from which all the e-commerce enterprises and individuals are worth learning.

\section{References}

[1] Liu Zhichao, Chen Yong, Yao Zhili. E-commerce service mode innovation in big data era [J]. Research on Science and Technology Management, 2014,01:31-34.

[2] Gao Xiaodong. Evaluation of e-commerce service mode in big data age [J]. Knowledge Economy, 2016,03: 64-66.

[3] Yang Tianhong. Discussion on data e-commerce service mode innovation in big data era [J]. Technology Information, 2016,24: 63-64.

[4] Jia Lifei. Research on e-commerce service mode in big data era [J]. Shopping Mall Modernization, 2014, 28: 105.

[5] Wang Huimin. Value creation and mode innovation of e-commerce under big data background [J]. Commercial Economics Research, 2015,07: 76-77.

[6] Chen Yuan, Liu Hui, Zhou Wenhao. Application of big data in Taobao e-commerce mode innovation [J]. Value Engineering, 2015,35: 1-5.

[7] Wang Zhongfeng. E-commerce service mode innovation in big data era [J]. E-commerce, 2017,03: 18-19. 